\title{
Assur decompositions of direction-length frameworks
}

\author{
Anthony Nixon
}

\begin{abstract}
A bar-joint framework is a realisation of a graph consisting of stiff bars linked by universal joints. The framework is rigid if the only bar-length preserving continuous motions of the joints arise from isometries. A rigid framework is isostatic if deleting any single edge results in a flexible framework. Generically, rigidity depends only on the graph and we say an Assur graph is a pinned isostatic graph with no proper pinned isostatic subgraphs. Any pinned isostatic graph can be decomposed into Assur components which may be of use for mechanical engineers in decomposing mechanisms for simpler analysis and synthesis. A direction-length framework is a generalisation of bar-joint framework where some distance constraints are replaced by direction constraints. We initiate a theory of Assur graphs and Assur decompositions for direction-length frameworks using graph orientations and spanning trees and then analyse choices of pinning set.
\end{abstract}

\section{Introduction}

A (bar-joint) framework $(G, p)$ in $\mathbb{R}^{d}$ is the combination of a finite graph $G=(V, E)$ and a map $p: V \rightarrow \mathbb{R}^{d} .(G, p)$ is rigid if the only edge-length-preserving continuous motions of the vertices arise from isometries of $\mathbb{R}^{d}$ and flexible if it is not rigid. It is typically of interest to characterise minimal rigidity, or isostaticity, which is when $(G, p)$ is rigid but $(G-e, p)$ is flexible for any $e \in E$.

In this article we will work with pinned frameworks where the locations of some subset of the vertex set are fixed in the framework; hence these points are completely immobilised. An Assur decomposition of an isostatic framework $(G, p)$ is a decomposition of the edge set of $G$ such that each component is rigid as a pinned framework and no subframework of any component has that property.

Anthony Nixon

Mathematics and Statistics, Lancaster University, LA1 4YF, U.K. e-mail: a.nixon@lancaster.ac.uk 
In mechanical engineering, analysis of isostatic graphs, often through Assur decompositions, is used in the design, synthesis and control of mechanisms $[2,16,18$, 19]. Mathematically, Assur decompositions of frameworks in $\mathbb{R}^{d}$ have been studied from combinatorial and geometric perspectives [13, 14, 17]. Of most relevance to us is the main result of [17] which shows that the Assur decomposition of a pinned isostatic graph is exactly equivalent, on the one hand, to a block decomposition of the pinned rigidity matrix and, on the other hand, to a strongly connected component decomposition of a $d$-orientation of the graph.

We extend these techniques to allow direction constraints. A DL-graph $G=$ $(V ; D, L)$ consists of a graph $G$ in which the edge set $E$ is partitioned into two parts $D$ and $L$. We refer to edges in $D$ as direction edges and edges in $L$ as length edges. A $d$-dimensional direction-length framework $(G, p)$, abbreviated henceforth to $D L$ framework, consists of a DL-graph $G=(V ; D, L)$ and a map $p: V \rightarrow \mathbb{R}^{d}$. (Throughout we will assume $d \geq 2$.) The framework has two types of constraint for the edges: each $e \in L$ will correspond to a length constraint; and each $f \in D$ to a direction constraint. We will say that a DL-framework is specifically one in which $D$ and $L$ are non-empty. We will use the terms: pure if either $D$ or $L$ is empty; length pure if $D=\varnothing$; and direction pure if $L=\varnothing$. Frameworks with direction constraints were first considered in [23]. Subsequently, in the 2-dimensional case [15], it was proved that for a graph $G=(V, E)$, a generic direction pure framework $(G, p)$ is rigid if and only if the corresponding length pure framework $(G, p)$ is rigid.

A DL-framework $(G, p)$ is generic if the set containing the coordinates of the vertices is algebraically independent over $\mathbb{Q}$. The following characterisation of rigidity for generic DL-frameworks in $\mathbb{R}^{2}$ was proved by Servatius and Whiteley.

Theorem 1 ([15]). A generic DL-framework $(G, p)$ is isostatic in $\mathbb{R}^{2}$ if and only if

1. $|D \cup L|=2|V|-2$;

2. $\left|E^{\prime}\right| \leq 2\left|V^{\prime}\right|-2$ for all $\left(V^{\prime}, E^{\prime}\right) \subset G$;

3. $\left|E^{\prime}\right| \leq 2\left|V^{\prime}\right|-3$ for all $\left(V^{\prime}, E^{\prime}\right) \subset G$ with $\left|E^{\prime}\right|>0$ and $E^{\prime} \subset D$ or $E^{\prime} \subset L$.

In Section 2 we provide further background on DL-frameworks and then we develop some basic results on pinned DL-frameworks. In Section 3 we define and characterise the Assur decomposition of a DL-framework. Section 4 discusses drivers. In particular we show which components of the Assur decomposition are in motion when a single edge is removed from a particular component. In Section 5 we look in more detail at the special case when the DL-framework is pinned with exactly 1 pinned vertex. In this case we describe how the Assur decomposition changes when we vary the choice of pinned vertex. We conclude, in Section 6, by discussing further avenues for exploration.

We expect Assur decompositions of pinned isostatic DL-frameworks will complement the existing uses of Assur decompositions in mechanical engineering $[18,19]$. Further applications may be possible in wireless sensor networks [3] or in the control of robotic formations [22, 24] where direction frameworks are often used under the name bearing rigidity. In particular, it may already be interesting to the bearing rigidity community to be aware that the Assur decomposition results of 
$[13,14,17]$ immediately adapt to the 2-dimensional direction pure case. In what follows we will work with DL-frameworks and develop analogous results.

\section{Pinned direction-length frameworks}

Two DL-frameworks $(G, p)$ and $(G, q)$ in $\mathbb{R}^{d}$ are said to be equivalent if $q(u)-q(v)$ is a scalar multiple of $p(u)-p(v)$ for all $u v \in D$ with $p(u) \neq p(v)$ and $\| p(u)-$ $p(v)\|=\| q(u)-q(v) \|$ for all $u v \in L$. They are congruent if $(G, q)$ can be obtained from $(G, p)$ by a translation and a dilation by \pm 1 . We say $(G, p)$ is rigid if there exists an $\varepsilon>0$ such that if a DL-framework $(G, q)$ is equivalent to $(G, p)$ and satisfies $\|p(v)-q(v)\|<\varepsilon$ for all $v \in V$ then $(G, q)$ is congruent to $(G, p)$. Equivalently, every continuous motion of the points $p(v), v \in V$ respecting the length and direction constraints results in a DL-framework which is congruent to $(G, p)$.

To introduce the rigidity matrix for a DL-framework, as in [5], take a DLframework $(G, p)$ in $\mathbb{R}^{d}$ where $p$ is injective. For any direction edge $e=u v$ we let $B_{e}$ be a $(d-1) \times d$ matrix whose rows are a basis for the subspace of $\mathbb{R}^{d}$ orthogonal to $\langle p(u)-p(v)\rangle$. A rigidity matrix $R_{D L}(G, p)$ for $(G, p)$ is a $((d-1)|D|+|L|) \times d|V|$ matrix constructed as follows. We first choose an arbitrary reference orientation for the edges of $D$, and use the notation $e=u v$ to mean that $e$ has been oriented from $u$ to $v$. Each edge in $D$ corresponds to $d-1$ consecutive rows of $R_{D L}(G, p)$, each edge in $L$ to one row of $R_{D L}(G, p)$, and each vertex in $V$ to $d$ consecutive columns of $R_{D L}(G, p)$. The submatrix of $R_{D L}(G, p)$ with rows labelled by $e=u v \in D$ and columns labelled by $x \in V$ is $B_{e}$ if $x=u$, is $-B_{e}$ if $x=v$, and is the $(d-1) \times d$ zero matrix otherwise. The submatrix of $R_{D L}(G, p)$ with row labelled by $e=u v \in L$ and columns labelled by $x \in V$ is $p(u)-p(v)$ if $x=u$, is $p(v)-p(u)$ if $x=v$, and is zero otherwise. It is easy to see that the kernel of $R_{D L}(G, p)$ always contains at least $d$ linearly independent vectors, corresponding to translations.

Next we introduce pinned frameworks with direction and length constraints. Let $G=(P, I ; D, L)$ consist of a graph $G$ on a vertex set $V$ which is partitioned into two parts $P$ and $I$, and an edge set $E$ which is also partitioned into two parts $D$ and $L$. We will consider rigidity where vertices in $P$ are pinned and vertices in $I$ are known as inner vertices.

Let $G=(P, I ; D, L)$ and $p: V \rightarrow \mathbb{R}^{d}$. In the DL-framework $(G, p)$ we have length and direction constraints as described above and each $v \in P$ is immobilised by any continuous motion. We say that $(G, p)$ is pinned rigid if every continuous motion of the points $p(v), v \in I$ respecting the length and direction constraints results in a DL-framework which is congruent to $(G, p)$. The rigidity matrix $R_{D L}^{\text {pin }}(G, p)$ for a pinned DL-framework $(G, p)$ in $\mathbb{R}^{d}$ arises from $R_{D L}(G, p)$ by deleting the $d$-tuple of columns corresponding to each pinned vertex $v \in P$. We define $(G, p)$ to be: pinned infinitesimally rigid if rank $R_{D L}(G, p)=d|I|$; pinned independent if $R_{D L}^{\mathrm{pin}}(G, p)$ has linearly independent rows; pinned isostatic if it is pinned infinitesimally rigid and pinned independent; and generic if the set of coordinates of the inner and pinned vertices is algebraically independent over $\mathbb{Q}$. We also say that (non-zero) vectors in 
$\operatorname{ker} R_{D L}^{\mathrm{pin}}(G, p)$ are infinitesimal motions of $(G, p)$. Hence infinitesimal motions only apply at inner vertices.

Remark 1. Note that in dimension greater than two each direction constraint provides more than 1 row in the rigidity matrix. Hence it is possible, simply for parity reasons, that a (pinned) direction pure framework is "minimally rigid" in the sense that it is rigid but deleting any edge results in a flexible framework, while at the same time having linearly dependent rows in its rigidity matrix. Since length edges provide precisely one row we can avoid this problem in the general case.

Example 1. Consider the graph $G=(P, I ; D, L)$ on 5 vertices where $P=\{v\}, G[I]$ induces the complete graph on 4 vertices, the edge set of $G[I]$ has been partitioned into two paths of length 3 one in $D$ and one in $L$, and the final two edges are incident to $v$ and to distinct points of $I$, again one each in $D$ and $L$. (See Figure 1.) The rigidity matrix, $R_{D L}^{\text {pin }}(G, p)$, of $(G, p)$ in $\mathbb{R}^{2}$ is as follows:

$$
\left(\begin{array}{cccc}
{\left[p\left(v_{1}\right)-p\left(v_{2}\right)\right]^{\perp}\left[p\left(v_{2}\right)-p\left(v_{1}\right)\right]^{\perp}} & 0 & 0 \\
p\left(v_{1}\right)-p\left(v_{3}\right) & 0 & p\left(v_{3}\right)-p\left(v_{1}\right) & 0 \\
{\left[p\left(v_{1}\right)-p\left(v_{4}\right)\right]^{\perp}} & 0 & 0 & {\left[p\left(v_{4}\right)-p\left(v_{1}\right)\right]^{\perp}} \\
0 & p\left(v_{2}\right)-p\left(v_{3}\right) & p\left(v_{3}\right)-p\left(v_{2}\right) & 0 \\
0 & p\left(v_{2}\right)-p\left(v_{4}\right) & 0 & p\left(v_{4}\right)-p\left(v_{2}\right) \\
0 & 0 & {\left[p\left(v_{3}\right)-p\left(v_{4}\right)\right]^{\perp}} & \left.p\left(v_{4}\right)-p\left(v_{3}\right)\right]^{\perp} \\
p\left(v_{1}\right)-p(v) & 0 & 0 & 0 \\
0 & {\left[p\left(v_{2}\right)-p(v)\right]^{\perp}} & 0 & 0
\end{array}\right),
$$

where you notice that the submatrix obtained by deleting the last two rows is precisely the matrix $R_{D L}\left(K_{4},\left.p\right|_{K_{4}}\right)$. It is not hard to check, for any generic $p \in \mathbb{R}^{2|P \cup I|}$, that rank $R_{D L}^{\text {pin }}(G, p)=8=|D|+|L|$ and hence $(G, p)$ is pinned isostatic in $\mathbb{R}^{2}$.

Any generic realisation of the graph $H$ is also pinned isostatic which is easy to deduce from the fact that $(G, p)$ is pinned isostatic as $H$ is formed from $G$ by a sequence of degree 2 vertex additions.

Note that it is easy to see that both examples are rigid, though not isostatic, when re-interpreted as pure frameworks. To obtain an example that is pinned iostatic as a DL-framework but flexible as a pure framework is also easy. One example would be to add four new vertices to $G$, add a $K_{4}$ on these new vertices and attach them to $G$ by two edges, say incident to $v_{3}$ and $v_{4}$. That the result will be pinned-isostatic follows from Theorem 1 (provided the new $K_{4}$ contains edges of each type), see also Proposition 1 below, but the framework has an obvious motion as a pure framework.

Jackson and Keevash [5] proved that, generically, rigidity and infinitesimal rigidity coincide for unpinned DL-frameworks and hence rigidity depends only on the underlying graph. Their techniques extend to pinned DL-frameworks giving us the following lemmas.

Lemma 1. Let $(G, p)$ be a generic pinned DL-framework. Then $(G, p)$ is rigid if and only if it is infinitesimally rigid. 

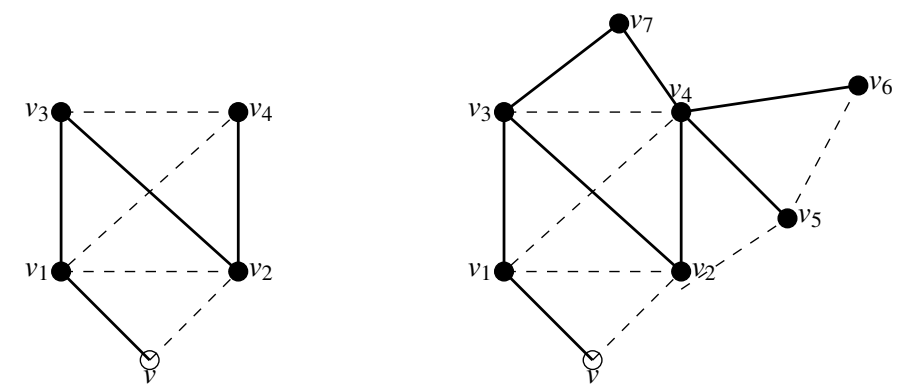

Fig. 1 Two pinned isostatic DL-frameworks $G, H$. We adopt the convention throughout that vertices in $P$ will be represented by unfilled circles and vertices in $I$ by filled circles. Furthermore, edges in $D$ will be represented by dashed lines and edges in $L$ by unbroken lines.

Lemma 2. Let $(G, p)$ be a generic pinned isostatic DL-framework in $\mathbb{R}^{d}$. Then $G$ satisfies

(1) $(d-1)|D|+|L|=d|I|$;

(2) $(d-1)\left|D^{\prime}\right|+\left|L^{\prime}\right| \leq d\left|I^{\prime}\right|$ for all $\left(V^{\prime}, E^{\prime}\right) \subset G$ with $V^{\prime}=P^{\prime} \cup I^{\prime}$ and $E^{\prime}=D^{\prime} \cup L^{\prime}$.

In fact, one may easily derive more precise necessary counts by considering the number of pinned vertices in a subgraph and whether it is pure or not. Indeed in $\mathbb{R}^{2}$ there are the following 3 additional conditions:

(3) $\left|D^{\prime}\right|+\left|L^{\prime}\right| \leq 2\left|I^{\prime}\right|-1$ for all pure subgraphs with $\left|P^{\prime}\right|=1$;

(4) $\left|D^{\prime}\right|+\left|L^{\prime}\right| \leq 2\left|I^{\prime}\right|-2$ for all subgraphs with $\left|P^{\prime}\right|=0$;

(5) $\left|D^{\prime}\right|+\left|L^{\prime}\right| \leq 2\left|I^{\prime}\right|-3$ for all pure subgraphs with $\left|P^{\prime}\right|=0$.

We note the following converse to Lemma 2 in dimension 2.

Proposition 1. Let $G=(V, E)$. A DL-framework $(G, p)$ is pinned isostatic in $\mathbb{R}^{2}$ if and only if $G$ satisfies conditions (1)-(5).

Proof. Necessity was discussed above. We prove that if $G$ satisfies (1)-(5) then $(G, p)$ is a pinned isostatic DL-framework. To do this we add a (non-pinned) isostatic DL-graph $G_{P}=\left(P, E_{P}\right)$ on the pinned vertices and then replace each pinned vertex with an inner vertex to get a graph $G^{*}=\left(V^{*}, E^{*}\right)$ where $V^{*}=V=I \cup P$ and $E^{*}=D^{*} \cup L^{*}=E \cup E_{P}$. Observe that, by (1) and by applying Theorem 1 to $G_{P}, G^{*}$ satisfies

$$
\left|E^{*}\right|=|E|+\left|E_{P}\right|=2|I|+2|P|-2=2\left|V^{*}\right|-2,
$$

and similarly, using (2)-(5) we have $\left|E^{\prime}\right| \leq 2\left|V^{\prime}\right|-2$ for all $\left(V^{\prime}, E^{\prime}\right) \subset G^{*}$ and $\left|E^{\prime}\right| \leq$ $2\left|V^{\prime}\right|-3$ for all $\left(V^{\prime}, E^{\prime}\right) \subset G^{*}$ with $E^{\prime} \subset D^{*}$ or $E^{\prime} \subset L^{*}$. Hence Theorem 1 implies that $\left(G^{*}, p\right)$ is (unpinned) isostatic. It follows that $(G, p)$ is isostatic.

In a DL-framework $(G, p)$ in $\mathbb{R}^{d}$, each direction constraint produces $d-1$ rows in $R_{D L}^{\mathrm{pin}}(G, p)$. Hence it will be convenient to consider the graph $G^{+}=\left(V^{+}, E^{+}\right)=$ 
$\left(P^{+}, I^{+} ; D^{+}, L^{+}\right)$which arises from $G$ by replacing each $e \in D$ with $d-1$ copies of the edge $e$ (and setting $P^{+}=P, I^{+}=I$ and $L^{+}=L$ ). Note in dimension 2, $G=G^{+}$. A $D L$-orientation of $G^{+}$is an orientation such that: for each edge of $D$ all parallel copies in $D^{+}$have the same orientation; all inner vertices have out-degree $d$; and all pinned vertices have out-degree 0 .

Lemma 3. Let $(G, p)$ be a pinned isostatic DL-framework in $\mathbb{R}^{d}$. Then there is a $D L$-orientation of $G^{+}$. Moreover let $\mathscr{O}$ and $\mathscr{O}^{\prime}$ be two DL-orientations of $G^{+}$. Then the strongly connected components are the same in both DL-orientations.

Proof. For any subgraph $\left(P^{\prime}, I^{\prime} ; D^{\prime}, L^{\prime}\right)$ of $G$ we have, By Lemma $2,\left|\left(D^{\prime}\right)^{+}\right|+$ $\left|\left(L^{\prime}\right)^{+}\right|=(d-1)\left|D^{\prime}\right|+\left|L^{\prime}\right| \leq d\left|I^{\prime}\right|$. The first assertion now follows from a standard result on orientations of sparse graphs first proved by Hakimi [4, Theorem 2]. The second conclusion is a consequence of the fact that $\mathscr{O}$ may be obtained from $\mathscr{O}^{\prime}$ by reversing directions on the edges in some set of cycles [17, Corollary 2.2].

\section{Assur graphs and Assur decompositions}

For a pinned DL-framework we can consider the minimal pinned isostatic subframework. This corresponds to the smallest subframework of $(G, p)$ which is pinned isostatic (necessarily this subframework contains at least one pinned vertex $v_{1}$ ). The edge set of such a subgraph is the first Assur component $C_{1}$. With $C_{1}$ chosen we consider a new graph in which the entire subgraph induced by $C_{1}$ is pinned. We then find the smallest pinned isostatic subframework and call it's edge set $C_{2}$. By repeating until $C_{1}, C_{2}, \ldots, C_{t}$ partitions the edge set of $G$ we obtain the Assur decomposition of $G$. It would be equivalent (see Proposition 2) to, at each stage, contract $C_{i}$ to a single pinned vertex. We can decompose the (square) pinned rigidity matrix into indecomposable blocks by permuting rows and columns until the blocks are in lower triangular form. Given a DL-orientation we can read off the strongly connected components of $G^{+}$. We augment the definition of strongly connected component by including edges directed out of the component.

Lastly, we use $d$-tree decomposition for the decomposition of $G^{+}$into components, each of which is the edge-disjoint union of $d$ spanning trees and no proper subgraph has that property. We insist that the first component contains some number of pinned vertices (and for the purpose of the tree decomposition, the pinned vertices are considered as a single vertex), and in subsequent components the earlier components are considered as a single pinned vertex. Given a graph that is the edgedisjoint union of $d$ spanning trees, a DL-orientation can be assigned. In particular we can choose $P$ as the sink (with out-degree 0 ) and direct the edges in each spanning tree towards $v$. Hence one may think of this decomposition as into edge-disjoint spanning trees along with edges directed out of the component.

Our first main result shows that these four decompositions are equivalent providing multiple ways of understanding, testing and computing Assur decompositions of generically isostatic DL-frameworks. 
Theorem 2. Given a generic pinned isostatic DL-framework $(G, p)$ and any $D L$ orientation of $G^{+}$, the following are equivalent:

(1) the Assur decomposition of $(G, p)$;

(2) the strongly connected decomposition of $G^{+}$;

(3) the block decomposition of $R_{D L}^{\mathrm{pin}}(G, p)$;

(4) the d-tree decomposition of $G^{+}$.

Proof. Since the equivalence of (1), (2) and (3) can be proven by adapting the technique used in [11, Theorem 3] (or alternatively in [17, Theorem 3.5]) we are brief. Observe first that, since $(G, p)$ is pinned isostatic, every square submatrix is invertible and hence $(3) \Rightarrow(1)$ is immediate.

For $(1) \Rightarrow(2)$ let $G_{1}$ be the graph of the first Assur component. By Lemma 3 we may choose a DL-orientation of $G^{+}$. Suppose $G_{1}^{+}$contains a proper subgraph $H_{1}^{+}$ which is a strongly connected component of $G^{+}$containing some pinned vertex. If $\left|E\left(H_{1}^{+}\right)\right|<d\left|I\left(H_{1}^{+}\right)\right|$then counting edges in $G_{1}^{+}-H_{1}^{+}$(including edges between them) contradicts the fact that $\left(G_{1},\left.p\right|_{G_{1}}\right)$ is isostatic. Hence Lemma 2 implies that $\left|E\left(H_{1}^{+}\right)\right|=d\left|I\left(H_{1}^{+}\right)\right|$, contradicting the assumption that $G_{1}$ is an Assur graph.

To see $(2) \Rightarrow(3)$ suppose there are two or more strongly connected components. Then take the bottom component with its edges to the pinned vertices. In $R_{D L}^{\mathrm{pin}}(G, p)$ apply a permutation of rows and a permutation of column vertices to place these rows and columns at the top left of the matrix. The remaining matrix forms a second block to which we iterate this process giving the desired lower block triangular form.

$(1) \Rightarrow$ (4) follows from Lemma 2 using a classical theorem of Nash-Williams [9] and similarly we can deduce $(4) \Rightarrow(3)$ noting that $(G, p)$ is isostatic so $R_{D L}^{\mathrm{pin}}(G, p)$ has no linearly dependent submatrices.

The equivalence also holds at the level of components. That is, the first Assur component is exactly the first strongly connected component, the first component of the block decomposition of the pinned direction-length rigidity matrix and the first component of the $d$-tree decomposition.

Example 2. In Figure 1 we gave examples of pinned isostatic DL-frameworks $(G, p)$ and $(H, q)$ in $\mathbb{R}^{2}$. $G$ itself is an Assur graph so the Assur decomposition is trivial. $G$ occurs as a subgraph of $H$ and hence is the first component $C_{1}$ in the Assur decomposition $C_{1}, C_{2}, C_{3}, C_{4}$ of $(H, p)$. The remainder of the decomposition consists of single vertices attached to the below components. Note that $C_{2}$ could either be $v_{5}$ or $v_{7}$; they are incomparable in the induced partial order, however $v_{6}$ must come after $v_{5}$. In general the partial order of the Assur decomposition is unique, but there can be multiple different linear extensions.

For pure isostatic frameworks in 2-dimensions, it was proved in [13] that contracting the set of pinned vertices to a single vertex takes us from a pinned isostatic framework to a framework whose graph is a generic circuit: that is the graph induced by a circuit in the generic 2-dimensional rigidity matroid (see [1] for details on this class of graphs). We can give the following analogue for DL-frameworks in our next result. 
Proposition 2. Let $(G, p)$ be a pinned DL-framework in $\mathbb{R}^{2}$ with $\left|D^{\prime}\right|+\left|L^{\prime}\right| \leq 2\left|I^{\prime}\right|-$ 1 for all pure subgraphs. Suppose that $P=\left\{v_{1}, \ldots, v_{k}\right\}$. Let $G^{\prime}=G / P$ denote the graph formed by contracting $v_{1}, \ldots, v_{k}$ to a single pinned vertex $v$. Then $(G, p)$ is pinned isostatic if and only if $\left(G^{\prime}, p^{\prime}\right)$ is pinned isostatic, for any generic $p^{\prime}$.

Proof. It is easy to verify that $G$ satisfies conditions (1), (2), (4) and (5) of Proposition 1 if and only if $G^{\prime}$ does. That $G$ satisfies (3) if and only if $G^{\prime}$ does follows from the hypothesis that $\left|D^{\prime}\right|+\left|L^{\prime}\right| \leq 2\left|I^{\prime}\right|-1$ for all pure subgraphs of $G$.

For example, suppose $G$ is the pinned graph consisting of a cycle $C_{k}$, for $k \geq 3$, of inner vertices, $P$ consists of a set of $k$ vertices and the remaining edges form a perfect matching between $C_{k}$ and $P$ (with any pattern of direction and length edges that respects the hypotheses of the proposition). Note that Proposition 1 implies that generic realisations of $G$ and the wheel graph $W_{k}$ obtained from $G$ by contracting the $k$ pinned vertices are pinned isostatic in $\mathbb{R}^{2}$.

We conclude this section with an algorithmic remark. The pebble game [6], as extended in [8], can be used to efficiently assign a DL-orientation to $G$. It is not hard to extend this to check the pure subgraph conditions and hence determine whether a pinned DL-framework is pinned isostatic in $\mathbb{R}^{2}$. Moreover finding strongly connected components, and hence Assur components, can be done in linear time [20].

\section{Drivers and strongly Assur graphs}

Key to applications of Assur graphs in mechanical engineering is the control and synthesis of mechanisms $[16,19]$. Thus, in this section, we derive several results showing how knowledge of the Assur decomposition allows us to control the 1 degree of freedom motion which results from deleting a single edge from a pinned isostatic DL-framework.

Lemma 4. Let $(G, p)$ be a generic pinned isostatic DL-framework in $\mathbb{R}^{d}$. Suppose $C_{k}$ is an Assur component containing the edge e $\in D \cup L$. Then $(G-e, p)$ has a nontrivial continuous motion which is necessarily zero on all vertices in components below or incomparable to $C_{k}$.

Proof. Since $(G, p)$ is pinned isostatic, $(G-e, p)$ is not infinitesimally rigid and hence Lemma 1 implies that $(G-e, p)$ has a non-trivial continuous motion. By the definition of Assur decomposition all components below or incomparable to $C_{k}$ are pinned isostatic and hence are fixed by the motion.

An Assur graph is strongly Assur if the infinitesimal motion created by removing any edge has a non-trivial velocity at every inner vertex.

Lemma 5. Let $(G, p)$ be a generic pinned isostatic DL-framework in $\mathbb{R}^{2}$. Suppose each component in the Assur decomposition is strongly Assur and let $C_{k}$ be an Assur component containing the edge e. Then $(G-e, p)$ has a non-trivial continuous 
motion which is non-zero on all inner vertices in $C_{k}$ and all inner vertices in components above $C_{k}$ in the Assur decomposition.

Proof. As in Lemma 4 there is a non-trivial continuous motion of $(G-e, p)$. That this motion is non-zero on all vertices in $C_{k}$ and all vertices in components above $C_{k}$ in the Assur decomposition follows from Proposition 1 and the definitions of Assur decomposition and strongly Assur graph.

The following lemma will put a strong condition on the nature of continuous motions for DL-frameworks in $\mathbb{R}^{2}$.

Lemma 6. Let $(G, p)$ be a generic pinned isostatic DL-framework in $\mathbb{R}^{2}$. Then $(G, p)$ is Assur if and only if $(G, p)$ is strongly Assur.

Proof. One direction is obvious. For the converse, assume $G$ is an Assur graph. Delete an edge $e=a b$ and we have $|E(G-e)|=2|I(G-e)|-1$ by Lemma 2. This implies there is a non-trivial infinitesimal motion $u$ of $(G-e, p)$. Suppose $u(v)=0$ for some $v \in I$. Then $v$ is rigidly connected to the pinned vertices. Hence Proposition 1 implies that $v$ must be contained in a pinned subgraph $H$ with $|E(H)|=2|I(H)|$. Since $H$ contains at most one of $a, b$ we have $|I(H)|<|I(G)|$ contradicting the minimality of $G$.

We remark that the corresponding result with $d \geq 3$ is already false in the length pure case (see [17]) and similar examples can be constructed for DL-frameworks.

\section{Grounding isostatic DL-frameworks}

In this section we consider how to pin isostatic DL-frameworks. Note that Proposition 2 motivates us to focus our attention on pinning a single vertex. We consider how the choice of this pinned vertex affects the resulting Assur decomposition.

Lemma 7. Let $\mathscr{T}$ be a tree on $n$ vertices. Let $G$ be the DL-graph on $V(\mathscr{T})$ formed from doubling every edge in $E(\mathscr{T})$ and assigning one copy of each edge to $D$ and one to $L$. Then, after pinning any single vertex, $(G, p)$ is pinned isostatic in $\mathbb{R}^{d}$, for any generic $p$, and the Assur decomposition of $(G, p)$ has $n-1$ components.

Proof. Let $(G, p)$ be generic in $\mathbb{R}^{d}$. Since $\mathscr{T}$ is a tree it is easy to see that $G$ can be constructed by a sequence of degree 2 vertex additions starting at any vertex. Let $K_{1} \rightarrow G_{1} \rightarrow G_{2} \rightarrow \cdots \rightarrow G_{n}=G$ be any such sequence. By analysing each $R_{D L}\left(G_{i},\left.p\right|_{G_{i}}\right)$ we see that $\left(G_{i},\left.p\right|_{G_{i}}\right)$ is isostatic for each $1 \leq i \leq n$. The first conclusion is now clear and the second follows from the fact that any subtree of $\mathscr{T}$ induces an isostatic subframework.

At the other extreme we have the following lemma. 
Lemma 8. Let $(G, p)$ be a generic pinned isostatic DL-framework in $\mathbb{R}^{d}$ and suppose that any proper subgraph $H$ of $G^{+}$satisfies $|E(H)| \leq d|V(H)|-(d+1)$. Then the Assur decomposition of $(G, p)$ has precisely one component.

Proof. The hypothesis on $G^{+}$ensures that $(G, p)$ has no proper pinned rigid subframework. The result follows.

A special case, when $d=2$, is to take $G=(V, E)$ to be a generic circuit, with any non-trivial partition of $E$ (into $D$ and $L$ ), and identify some $v \in V$ as pinned.

Next, given an arbitrary pinned isostatic DL-framework, we consider how to determine which vertex is the optimal choice to pin in order to maximise, or minimise, the number of components. To answer this question, we introduce the following directed acyclic graph. Let $G=(V, E)$ and $(G, p)$ be a generic (unpinned) isostatic DL-framework in $\mathbb{R}^{d}$. We form a directed graph $\vec{D}=(U, F)$, which we shall call the pinning digraph for $G$ as follows. The set $U$ is the set of subsets of $V$ which induce unpinned isostatic graphs in $G$ (including $V$ itself and each single vertex). There is an edge directed from $X \in U$ to $Y \in U$ if and only if $X \subsetneq Y$ and there is no $Z \in U$ such that $X \subsetneq Z \subsetneq Y$.

Example 3. Let $(H, p)$ be the pinned isostatic DL-framework in Figure 1. Consider the (unpinned) isostatic framework $\left(H-v,\left.p\right|_{H-v}\right)$. In Figure 2 we construct the pinning digraph $\vec{D}$ for $\left(H-v,\left.p\right|_{H-v}\right)$. If we take $v_{6}$ as the pinned vertex then we obtain the Assur decomposition $C_{1}=K_{4}+\left\{v_{5}, v_{6}\right\}$ and $C_{2}=K_{4}+\left\{v_{5}, v_{6}, v_{7}\right\}$, whereas if we take $v_{3}$ as the pinned vertex then the Assur decomposition has alternative linear extensions of the partial order, one such choice being $C_{1}=K_{4}, C_{2}=K_{4}+v_{5}$, $C_{3}=K_{4}+\left\{v_{5}, v_{7}\right\}, C_{4}=K_{4}+\left\{v_{5}, v_{6}, v_{7}\right\}$.

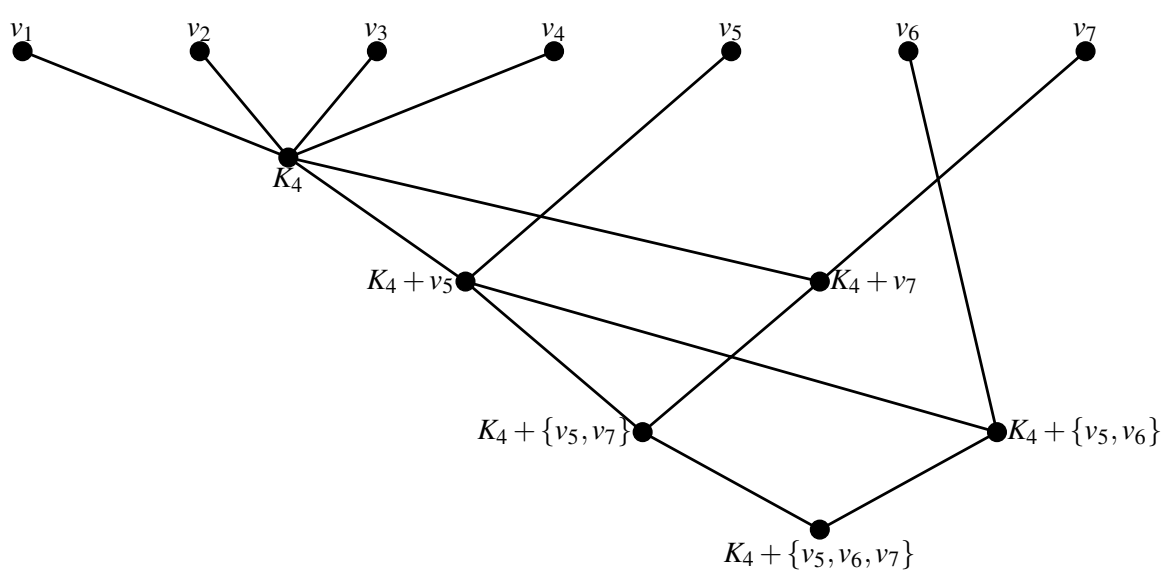

Fig. 2 A pinning digraph of an unpinned isostatic DL-framework. All edges are directed vertically downwards in the figure, arrows omitted. For brevity $K_{4}$ is used to denote its vertex set. 
The following lemma records some basic properties of pinning digraphs; each property follows quickly from the definition.

Lemma 9. Let $(G, p)$ be a generic isostatic DL-framework in $\mathbb{R}^{d}$ and let $\vec{D}$ be the pinning digraph for $G$. Then: $\vec{D}$ is acyclic and triangle-free; $V$ is the unique sink of $\vec{D}$; and each vertex $v \in V$ is a source of $\vec{D}$.

Theorem 3. Let $G=(V, E)$ and let $(G, p)$ be a generic isostatic DL-framework in $\mathbb{R}^{d}$. Then the Assur decomposition of $(G, p)$ with $x \in V$ pinned is in one-one correspondence with the set of directed paths from $x$ to $V$ in $\vec{D}$.

Proof. It follows from the construction of $\vec{D}$ that each path from $x$ to $V$ corresponds to a linear extension of the partial order of the Assur decomposition of $(G, p)$ with $x$ pinned. The theorem follows from the uniqueness of the partial order associated to an Assur decomposition of a pinned isostatic framework.

In particular, this implies that every directed path from $x$ to $V$ has the same length. Thus we can use $\vec{D}$ to choose a pinned vertex which will minimise, or maximise, the number of components in an Assur decomposition.

Corollary 1. Let $G=(V, E)$ and let $(G, p)$ be a generic isostatic DL-framework in $\mathbb{R}^{d}$ with pinning digraph $\vec{D}$. Let $x \in V$ be the source of $\vec{D}$ whose distance to $V$ is minimal (resp. maximal). Then pinning $x$ results in a pinned isostatic graph $G$ whose Assur decomposition has the minimum (resp. maximum) number of Assur components.

\section{Concluding remarks}

There are an array of open questions and potential extensions. We mention just three.

Complexity of the Assur decomposition. How may we minimise the complexity of the Assur decomposition? This could be in terms of the number of components, how close to linear the partial order can be or the complexity of individual components. We pose the question, given a random generic isostatic DL-framework in $\mathbb{R}^{2}$, what structure does the associated pinning digraph have?

Special positions of Assur graphs. For length pure frameworks, it was proved in [14] that any Assur graph has a special position in which there is a nowhere zero equilibrium stress ${ }^{1}$ and a special position in which there is a nowhere zero infinitesimal motion. It is not clear how to extend these results to DL-frameworks as their proof technique breaks down (see Proposition 2).

Alternative constraint systems. There are a number of other rigidity contexts where the count $|E|=k|V|-k$ is fundamental including: frameworks on the cylinder [10], in $\ell_{q}$ spaces [7], fixed lattice periodic frameworks [12] and body-bar frameworks [21]. We expect our techniques can be adapted to each of these contexts.

\footnotetext{
${ }^{1}$ An equilibrium stress is a vector in the cokernel of the rigidity matrix.
} 


\section{References}

1. Berg, A.R., Jordán, T.: A proof of Connelly's conjecture on 3-connected circuits of the rigidity matroid. J. Combin. Theory Ser. B 88(1), 77-97 (2003)

2. Durango, S., Correa, J., Ruiz, O.E.: Graph-based structural analysis of planar mechanisms. Meccanica 52(1-2), 441-455 (2017)

3. Eren, T.: Cooperative localization in wireless ad hoc and sensor networks using hybrid distance and bearing (angle of arrival) measurements. EURASIP Journal on Wireless Communications and Networking 2011(1), 72 (2011)

4. Hakimi, S.L.: On the degrees of the vertices of a directed graph. J. Franklin Inst. 279, 290-308 (1965)

5. Jackson, B., Keevash, P.: Bounded direction-length frameworks. Discrete Comput. Geom. 46(1), 48-71 (2011)

6. Jacobs, D.J., Hendrickson, B.: An algorithm for two-dimensional rigidity percolation: the pebble game. J. Comput. Phys. 137(2), 346-365 (1997)

7. Kitson, D., Power, S.C.: Infinitesimal rigidity for non-Euclidean bar-joint frameworks. Bull. Lond. Math. Soc. 46(4), 685-697 (2014)

8. Lee, A., Streinu, I.: Pebble game algorithms and sparse graphs. Discrete Math. 308(8), 14251437 (2008)

9. Nash-Williams, C.S.J.A.: Edge-disjoint spanning trees of finite graphs. J. London Math. Soc. 36, 445-450 (1961)

10. Nixon, A., Owen, J.C., Power, S.C.: Rigidity of frameworks supported on surfaces. SIAM J. Discrete Math. 26(4), 1733-1757 (2012)

11. Nixon, A., Schulze, B., Sljoka, A., Whiteley, W.: Symmetry adapted Assur decompositions. Symmetry 6(3), 516-550 (2014)

12. Ross, E.: Inductive constructions for frameworks on a two-dimensional fixed torus. Discrete Comput. Geom. 54(1), 78-109 (2015)

13. Servatius, B., Shai, O., Whiteley, W.: Combinatorial characterization of the Assur graphs from engineering. European J. Combin. 31(4), 1091-1104 (2010)

14. Servatius, B., Shai, O., Whiteley, W.: Geometric properties of Assur graphs. European J. Combin. 31(4), 1105-1120 (2010)

15. Servatius, B., Whiteley, W.: Constraining plane configurations in computer-aided design: combinatorics of directions and lengths. SIAM J. Discrete Math. 12(1), 136-153 (1999)

16. Shai, O.: Topological Synthesis of All 2D Mechanisms Through Assur Graphs. pp. 1727-1738 (2010)

17. Shai, O., Sljoka, A., Whiteley, W.: Directed graphs, decompositions, and spatial linkages. Discrete Appl. Math. 161(18), 3028-3047 (2013)

18. Sljoka, A., Shai, O., Whiteley, W.: Checking Mobility and Decomposition of Linkages via Pebble Game Algorithm. pp. 493-502 (2011)

19. Sun, Y., Ge, W., Zheng, J., Dong, D.: Solving the Kinematics of the Planar Mechanism Using Data Structures of Assur Groups. Journal of Mechanisms and Robotics 8(6) (2016). 061002

20. Tarjan, R.: Depth-first search and linear graph algorithms. SIAM J. Comput. 1(2), 146-160 (1972)

21. Tay, T.S.: La méthode de Henneberg appliquée aux charpentes de barres et de corps rigides. Structural Topology (17), 53-58 (1991). Dual French-English text

22. Trinh, M.H., Zhao, S., Sun, Z., Zelazo, D., Anderson, B.D.O., Ahn, H.S.: Bearing-based formation control of a group of agents with leader-first follower structure. IEEE Trans. Automat. Control 64(2), 598-613 (2019)

23. Whiteley, W.: Some matroids from discrete applied geometry. In: Matroid theory (Seattle, WA, 1995), Contemp. Math., vol. 197, pp. 171-311. Amer. Math. Soc., Providence, RI (1996)

24. Zhao, S., Zelazo, D.: Bearing rigidity theory and its applications for control and estimation of network systems: life beyond distance rigidity. IEEE Control Syst. 39(2), 66-83 (2019) 